\title{
Refractive Index Formula of Blood as a Function of Temperature and Concentration
}

\author{
MURAT ODUNCUOĞLU
}

\begin{abstract}
The analysis of blood parameters is main procedure for defining the patient's condition. The refractive index of blood was calculated using the experimental data for a medical application and diagnostic purposes. There is small change in the refractive indices with changing with the personal conditions, illness, parasitization, temperature and others. Theoretical simulations of the refractive index of blood are difficult, and it is unpredictable in different conditions. We proposed a new formula for the refractive index of blood as a function of wavelength, concentration, and temperature by using the genetic programming method. Input parameters were temperature $\left({ }^{\circ} \mathrm{C}\right)$, concentration $(\mathrm{g} / \mathrm{L})$, and wavelength $(\mathrm{nm})$. The refractive index values of blood were the output parameters. A total of 492 training and testing sets were selected in the spectral range of 436 to 1550 $\mathrm{nm}$, in the temperature range of $20-45^{\circ} \mathrm{C}$ and the concentration of $\mathrm{o}-200 \mathrm{~g} / \mathrm{L} \mathrm{HbC}$. The model proposes the refractive index formula blood for all the input parameters given in the range without need of extra parameters. The results are good agreement with experimental measurements in the literature and compared to Sellmeier equation.
\end{abstract}

Key words: Artificial intelligence, refractive index, human blood, genetic programming.

\section{INTRODUCTION}

The optical properties of blood provide important information for diagnostic applications. The health care professionals have looked for medical conditions and signs of disease in the blood parameters. This connective liquid within the body are used to determine the condition with non-invasively. The cells are suspended in plasma of heterogeneous medium of blood. Blood plasma contains water and various water-soluble proteins and low molecular compounds (Tuchin 2015). The blood cells consist of erythrocytes, leukocytes, and platelets with different ratios and sizes depending on demographic properties and diet (Friebel et al. 2006). The determination of refractive index of blood is attractive and have important applications involve sensing of blood groups (Rakhshani 2020, Zhernovaya et al. 2011), oxygenated and deoxygenated hemoglobin samples (Alade et al. 2018), optical diagnosis and laser treatments (Jin et al. 2006) and other applications. Researchers have proposed different methods for deriving the real and complex part of the refractive index (Oduncuoğlu 2020, Tuchin 2015, 2016, Zhernovaya et al. 2011). The refractive index is a defined as $n=n_{\text {Real }}+n_{\text {Imaginary }}$. The $n=n_{\text {real }}$ is the real part and $n=n_{\text {Imaginary }}$ is the imaginary part of complex number, respectively. The real part represents the change in the speed of propagated light through a medium, compared to the speed of light in a vacuum and an imaginary part is the related to absorption parameter of material. The structure of blood is complex, and this attractive research subject is mostly studied to develop 
a mathematical model. The optical parameters of blood depend on the optical properties of its components. Blood carries oxygen and nutrients to the trillions of cells is a very complex medium, which makes the theoretical simulation of its properties considerably difficult. In a simplified model, the blood can be presented as (Heller 1965, Tuchin 2016)

$$
n=\sum_{i=1}^{N} n_{i} f_{i}
$$

where $n_{i}$ and $f_{i}$ are the refractive indices and the volume fractions of $\mathrm{N}$ individual components of cells suspended in plasma, respectively. In this study, we mainly focus on real part of refractive index values of blood. The optical properties of blood were different from the other human tissues (Lazareva \& Tuchin 2018). The optical properties of cells and blood are determined both experimentally and theoretically to interpret their interaction with light. Artificial intelligence modeling is used to determine parameters and solve the unknown mechanism of the systems. Al aims to generate results with high accuracy to simulate the data. The main role of this program is the use of information (Kurt \& Oduncuoğlu 2015a, b, Kurt et al. 2015, Verdegay et al. 2008, Yilmaz et al. 2018). An important trend for the future of health technology will be the increasing use of artificial intelligence for medical applications with increased efficiency and effectiveness. The fact that the intelligence of healthcare in the future must come both from artificial and conventional forms (Mitchell \& Haroun 2016). The new medical technologies are developed by using this infrastructure. The artificial intelligence in healthcare has the potential to save costs, broadening accessibility, and can be an asset for patients and providers. We proposed a formula to determine the refractive index of blood by using genetic programming. Genetic programing uses the given experimental data to find desired parameter of given problems (Yilmaz et al. 2018). The genetic programming as a powerful modeling technique related to the statistical approach seems very logical method for predicting the parameter (Koza 1992).

\section{EXPERIMENTAL SETUP}

The optical properties of blood depend on physiological and biochemical parameters are determined. The experimental data for the refractive index of blood are measured using the Abbemat Refractometer (Friebel et al. 2006, Jacques 1965, Lazareva \& Tuchin 2018, Liu et al. 2019, Meinke et al. 2006, Nahmad-Rohen et al. 2015, Yahya \& Saghir 2016). The collected experimental data in papers were conducted by using dry human hemoglobin Lyophilized powder dissolved in isotonic phosphate-buffered saline to maintain the PH at 7.4 (Sydoruk et al. 2012, Yahya \& Saghir 2016, Zhernovaya et al. 2011). The real part of the refractive index of blood were modelled and estimated based on the concentration, temperature, and wavelength. The proposed model was developed using genetic programming. The proposed model is able to predict the refractive index of blood with an acceptable level of accuracy.

\section{THE MODEL}

Recently, it is common to use computers and computational methods to solve problems. In this study, the genetic programming for the formulation of the refractive index is used. The input parameters are given in Table I. 
Table I. The variables used in model.

\begin{tabular}{|l|l|l|r|}
\hline Input Variable & Range & Output Variable & Range \\
\hline Wavelength & $430-1550 \mathrm{~nm}$ & & \\
Temperature & $20-45^{\circ} \mathrm{C}$ & Refractive Index & $1.321-1.395$ \\
Concentration & $0-200 \mathrm{~g} / \mathrm{L}$ & & \\
\hline
\end{tabular}

The thermal and concentration dependence of the refractive index varies with wavelength. There are different formulations for determining the refractive index of blood, but no direct explicit formulations related to temperature, concentration, and wavelength. These ranges show the potential for clinical applications. The formulations were proposed by genetic programming to automatically extract relationships in a system for predicting the refractive index. The genetic programming main goal is to solve problems by searching all possible solutions. A genetic programming is adapted from the Darwinian evolution theory of natural selection based on survival of the fittest resulting from variability crossing over and mutation phenomena. The parameters in this system given in Table II are the chromosomes and expression trees. The simple rules determine the spatial organization of the functions and terminals in the expression trees and the type of interaction between sub-expression trees (Ferreira 2006). The iterative computational process is used to find successive generations for fitness until the fittest solutions are obtained.

GP is used for predicting parameter tool in medicine, social sciences, and other disciplines. The genetic programming are explained in books and papers (Ferreira 2006, Goldberg \& Holland 1998, Koza 1992, 1994, Koza \& Koza 1992). It was aimed to be in the best fitness zone, to minimize errors, to simplify, and to find the best predicted result (Pouraliakbar et al. 2016). A combination of these were investigated the increase performance of the algorithm in predicting output parameter. The optimal simplified equation for refractive index equation is given below:

$$
n_{\text {real }}=\left[1.557037+\frac{T}{\lambda(5.013366+C))}\right]^{\frac{2}{3}}+\frac{-29.07134(C+9.98566)}{\lambda^{2}}+\frac{6.34024-T}{6.902436^{2}(C+\lambda)}
$$

where $\mathrm{C}$ is the concentration $(\mathrm{g} / \mathrm{L})$, $\mathrm{T}$ is the temperature $\left({ }^{\circ} \mathrm{C}\right)$, and $\lambda$ is the wavelength $(\mathrm{nm})$. The refractive index of blood with different parameters $n_{\text {Real }}(T, C, \lambda)$ has been calculated by using this equation. The accuracy of the model was evaluated using statistical parameters. The coefficient of correlation, the root mean square error (RMSE) and the mean absolute error (MAE) and explanations of these parameters have been provided in detail References (Kurt \& Oduncuoğlu 2015b, Kurt et al. 2016). The correlation coefficient ( $R$ ) was used to calculate the model performance. The calculated results were in good agreement with the experimental results.

\section{RESULTS AND DISCUSSION}

The generalization performance of genetic programming by comparing the experimental and the predicted values are given in Figures 1-2. It is clearly observed that the formulation proposed by 
Table II. Genetic Programming parameters used for proposed models.

\begin{tabular}{|l|l|}
\hline Name & Data \\
\hline Number of Generations & 251511 \\
Chromosomes & $+^{\prime},-,,, I, \sqrt{x}, \exp (x), x^{2}, x^{3}, x^{1 / 3}$ \\
Head Size & 30 \\
Number of Genes & 7 \\
Training Samples & 3 \\
Testing Samples & 445 \\
Linking Function & 47 \\
Training $R^{2}$ & Addition \\
Testing $R^{2}$ & 0.9499 \\
Fitness Function & 0.9434 \\
Mean Squared Error (MSE) & RRSE \\
Mean Absolute Error (MAE) & $3.3810^{-6}$ \\
Relative Absolute Error (RAE) & $1.4310^{-3}$ \\
Root Mean Squared Error (RMSE) & $2.0810^{-1}$ \\
Correlation Coefficient & $1.8410^{-3}$ \\
\hline
\end{tabular}

the genetic programming formula for the refractive index is very successful. The Sellmeier equation is used to compare the results of our predicted data and is given in Equation 3 as

$$
n^{2}(\lambda)=1+\frac{A_{1} \lambda^{2}}{\lambda^{2}-B_{1}}+\frac{A_{2} \lambda^{2}}{\lambda^{2}-B_{2}}
$$

The empirically determined coefficients of the Sellmeier equation for whole blood are listed in Table III.

Table III. The coefficients of Sellmeier equations of blood (Liu et al. 2019).

\begin{tabular}{|l|l|l|l|}
\hline$A_{1}$ & $A_{2}$ & $B_{1}$ & $B_{1}$ \\
\hline 0.7960 & 5.1819 & $1.077210^{4}$ & $-7.830110^{5}$ \\
\hline
\end{tabular}

The wavelength dependence of the refractive index of water is determined by the expression (Kohl et al. 1994).

$$
n_{\text {water }}(\lambda)=1.31848+\frac{6.662}{\lambda-129.2}
$$




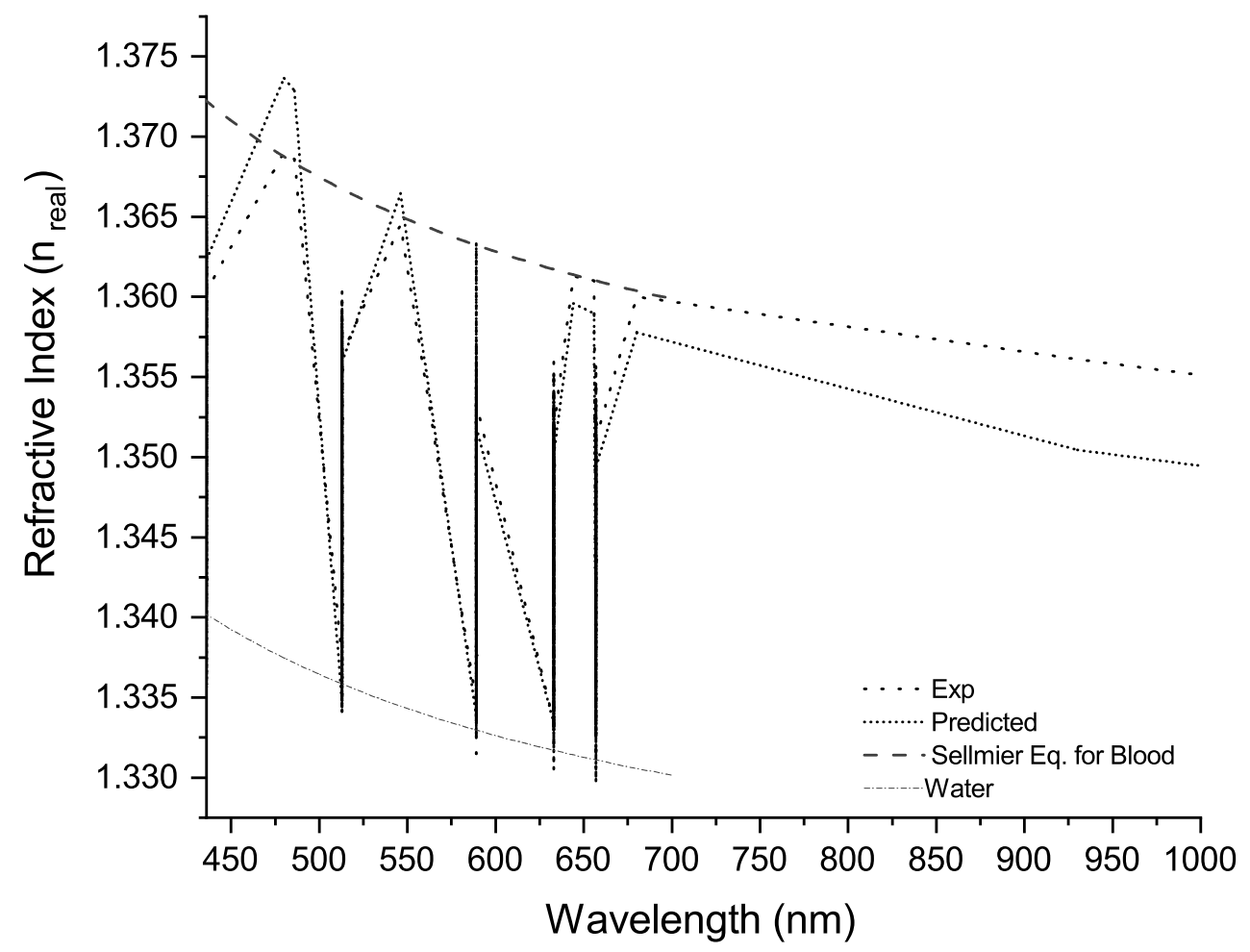

Figure 1. The experimental and calculated refractive index of blood $n_{\text {refractive }}(T, C, \lambda)$ with different temperatures $(T)$ and concentration (C).

The wavelength dependences of the refractive index were calculated by using the proposed formula and compared with refractive index values of blood calculated using Sellmeier equations and water obtained from Equation 4. These equations just depend on wavelength. The blood plasma contains up to $90 \%$ water.

The proposed equation is in good agreement with experimental and calculated results. The refractive index is increasing with increasing concentration (the blood contains low water) and decreasing with increasing temperature accordingly. The average values of temperature rate is

$$
\frac{d n_{\text {real }}}{d T}=-1.0510^{-4}\left(1{ }^{\circ} \mathrm{C}\right)
$$

and the average value of concentration is

$$
\frac{d n_{\text {real }}}{d C}=1.5410^{-4}(\mathrm{~g} / \mathrm{L})
$$

The proposed formula is more sensitive in trend but at low wavelength range, there is a small difference between experimental to predicted data. The linear model is obtained using the following equations

$$
n_{\text {refractive }}=n_{\text {real }}(\lambda)+\frac{d n_{\text {real }}}{d T} T\left({ }^{\circ} C\right)+\frac{d n_{\text {real }}}{d C} C(g / L)
$$

The non-linear behavior variations in refractive indices concerning wavelength were observed. The refractive index increases in a non-linear fashion as the wavelength decreases. This is due to electromagnetic interactions between the particles of light and particles within the blood. 


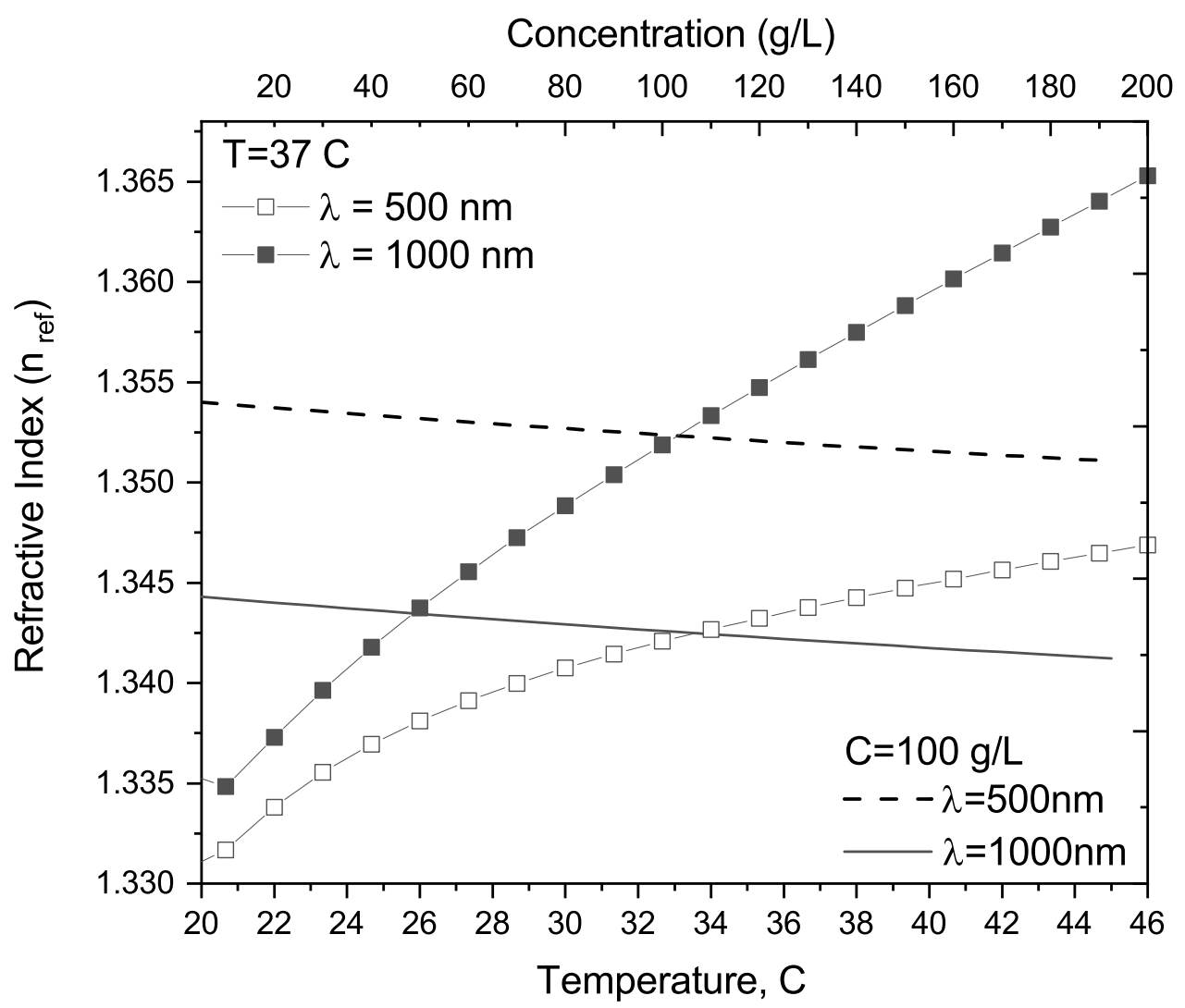

Figure 2. The calculated refractive index of blood as a function of temperature and concentration for wavelengths of 500 and $1000 \mathrm{~nm}$. The former is in the visible and the latter is in the near-infrared spectral range of the electromagnetic spectrum. The new model validated within these wavelength ranges and the trends are shown in the graph.

\section{CONCLUSION}

In this study, we proposed a new formulation for predicting the refractive index of blood as a function of wavelength, low concentration of protein, and temperature using genetic programming. The genetic programming works well and provides optimized parameters of real part of refractive index for blood. The formula transforms the experimental results easily to the computational media, making them useful for computational simulation of refractive index of blood used in the medical sciences. The refractive index formula is across a wide range of hemoglobin concentrations, temperatures, and wavelengths. It gives us an opportunity to compare all the parameters with each other. The predictive capacity of the program is $98.73 \%$ of the training and $98.37 \%$ of the testing results. The proposed equations are user-friendly. The statistical parameters $\left(R^{2}\right.$, MAPE, and RMS) of the proposed formulation show high potential for predicting the refractive index and use the results for diagnostic purposes. In this study, a simple linear refractive index formula with respect to parameters are also developed and analyzed. The proposed model used key parameters, such as the concentrations, temperature, and wavelength to accurately estimate the refractive index from experimental data. 


\section{REFERENCES}

ALADE IO, BAGUDU A, OYEHAN TA, ABD RAHMAN MA, SALEH TA \& OLATUNJI SO. 2018. Estimating the refractive index of oxygenated and deoxygenated hemoglobin using genetic algorithm-support vector regression model. Biomed Comput Meth Prog Bio 163: 135-142.

FERREIRA C. 2006. Gene expression programming: mathematical modeling by an artificial intelligence Vol. 21, Springer.

FRIEBEL M, ROGGAN A, MÜLLER GJ \& MEINKE MC. 2006. Determination of optical properties of human blood in the spectral range 250 to $1100 \mathrm{~nm}$ using Monte Carlo simulations with hematocrit-dependent effective scattering phase functions. J Biomed Opt 11(3): 0340212.

GOLDBERG DE \& HOLLAND JH. 1998. Genetic algorithms and machine learning. 1st ed. Kluwer Academic Publishers.

HELLER W. 1965. Remarks on refractive index mixture rules. J Phys Chem 69(4): 1123-1129.

JACQUES SL. 1965. Optical properties of biological tissues: a review. Phys Med Biol 58(11): R37-R61. DOI:10.1088/0031-9155/58/11/r37.

JIN Y, CHEN J, XU L \& WANG P. 2006. Refractive index measurement for biomaterial samples by total internal reflection. Phys Med Biol 51(20): N371.

KOHL M, COPE M, ESSENPREIS M \& BÖCKER D. 1994. Influence of glucose concentration on light scattering in tissue-simulating phantoms. Opt Lett 19(24): 2170-2172.

KOZA JR. 1992. Evolution of subsumption using genetic programming. Proceedings of the First European Conference on Artificial Life 1: 21.

KOZA JR. 1994. Genetic algorithms and machine learning, Genetic programming II (Vol. 17). MIT Press Cambridge.

KOZA JR \& KOZA JR. 1992. Genetic programming: on the programming of computers by means of natural selection (Vol. 1). MIT Press Cambridge.

KURT HI \& ODUNCUOĞLU M. 2015a. Effects of temperature, time, magnesium, and copper on the wettability of Al/TiC system. Math Probl Eng 710526: 21.

KURT HI \& ODUNCUOĞLU M. 2015b. Formulation of the effect of different alloying elements on the tensile strength of the in situ Al-Mg2Si composites. Metals 5(1): 371-382.

KURT HI, ODUNCUOĞLU M \& ASMATULU R. 2016. Wear behavior of aluminum matrix hybrid composites fabricated through friction stir welding process. J Iron Steel Res Int 23(10): 1119-1126.

KURT HI, ODUNCUOĞLU M \& KURT M. 2015. A mathematical formulation to estimate the effect of grain refiners on the ultimate tensile strength of $\mathrm{Al}-\mathrm{Zn}-\mathrm{Mg}-\mathrm{Cu}$ alloys. Metals 5(2): 836-849.

LAZAREVA EN \& TUCHIN VV. 2018. Blood refractive index modelling in the visible and near infrared spectral regions. J Biomed Photonics Eng 4(1): 1.

LIU S ET AL. 2019. Measurement of the refractive index of whole blood and its components for a continuous spectral region. J Biomed Opt 24(3): 035003. doi:10.1117/1.JBO.24.3.035003.

MEINKE M, FRIEBEL M, LUTTER A, GERSONDE I \& ROGGAN A. 2006. Determination of optical properties of human blood to develop new devices for blood monitoring. J Biomed Opt 12(1): 1-9.

MITCHELL D \& HAROUN L. 2016. Introduction to Health Care. 1st ed. Cengage Learning.

NAHMAD-ROHEN A, CONTRERAS-TELLO H, MORALES-LUNA $G$ \& GARCÍA-VALENZUELA A. 2015. On the effective refractive index of blood. Phys Scr 91(1): 015503.

ODUNCUOĞLU M. 2020. Optical Properties of Dilute Bismuth of Semiconductor Alloys. ECJSE 7(3): 1355-1361.

POURALIAKBAR H, FIROOZ S, JANDAGHI MR, KHALAJ G \& NAZARI A. 2016. Predicting the ultimate grain size of aluminum sheets undergone constrained groove pressing. Int J Adv Manuf Syst 86(5-8): 1639-1658.

RAKHSHANI MR. 2020. Optical refractive index sensor with two plasmonic double-square resonators for simultaneous sensing of human blood groups. Photonic and Nanostruct-Fundamentals and Applications 39: 100768.

SYDORUK O, ZHERNOVAYA OS, TUCHIN VV \& DOUPLIK A. 2012. Refractive index of solutions of human hemoglobin from the near-infrared to the ultraviolet range: Kramers-Kronig analysis. J Biomed Opt 17(11): 115002.

TUCHIN VV. 2015. Tissue Optics. Society of Photo-Optical Instrumentation Engineers (SPIE).

TUCHIN VV. 2016. Handbook of Optical Biomedical Diagnostics: Methods. Society of Photo-Optical Instrumentation Engineers (SPIE).

VERDEGAY JL, YAGER RR \& BONISSONE PP. 2008. On heuristics as a fundamental constituent of soft computing. Fuzzy Sets Syst 159(7): 846-855. 
YAHYA M \& SAGHIR M. 2016. Empirical modelling to predict the refractive index of human blood. Phys Med Biol 61(4): 1405.

YILMAZ NF, KURT HI, ODUNCUOĞLU M \& ERGUL E. 2018. Experimental and theoretical analysis of the welding process parameters for UTS with different methods. Mater Res Express 6(1): 016524.

ZHERNOVAYA O, SYDORUK O, TUCHIN V \& DOUPLIK A. 2011. The refractive index of human hemoglobin in the visible range. Phys Med Biol 56(13): 4013.

\section{How to cite}

ODUNCUOĞLU M. 2021. Refractive Index Formula of Blood as a Function of Temperature and Concentration. An Acad Bras Cienc 93: e20201634. DOI 10.1590/o001-3765202120201634.

Manuscript received on October 15, 2020;

accepted for publication on September 25, 2021

\section{MURAT ODUNCUOĞLU1,2}

https://orcid.org/0000-0002-3130-5646

${ }^{1}$ Technical Sciences VHS, Gaziantep University, 27310, Gaziantep, Turkey

${ }^{2}$ Yildiz Technical University, Science and Arts Faculty, Physics Department, 34220, Istanbul, Turkey

E-mail:murat@gantep.edu.tr,murato@yildiz.edu.tr 